\title{
A STUDY ON CLINICAL PROFILE OF PATIENTS WITH HIRSUTISM
}

\author{
Kunjumani Sobhanakumari', Thomas Reshma²
}

1 Professor and HOD, Department of Dermatology, Government T. D. Medical College, Alappuzha, Kerala. 2Junior Consultant, Department of Dermatology, Kochi.

\section{ABSTRACT}

\section{BACKGROUND}

Hirsutism is the presence of terminal hair in male like pattern in females in prepubertal and adult life. It affects $5-10 \%$ of women in childbearing age. However, hirsutism has poorly been studied in Indian patients.

Aim- To study the clinical profile of patients with hirsutism and to detect its various causes.

\section{MATERIALS AND METHODS}

In this observational cross sectional study, 60 consecutive cases of hirsutism attending the Dermatology and Endocrinology outpatient wings of Govt. Medical College, Kottayam were included. All underwent detailed clinical examination including modified Ferriman-Gallwey scoring and transabdominal ultrasound. Serum total testosterone, dehydroepiandrosterone sulfate (DHEAS), 17-hydroxyprogesterone (17-OHP), prolactin, free tri-iodothyronine, free tetra-iodothyronine, and thyroid stimulating hormone, fasting blood glucose and lipid profile were estimated.

\section{RESULTS}

The commonest cause of hirsutism was polycystic ovary syndrome (PCOS) in 42 (70\%) cases, followed by idiopathic hirsutism (IH) in $16(26.7 \%)$. There was $1(1.7 \%)$ case each of non-classic congenital adrenal hyperplasia (NCCAH) and acromegaly with pituitary macroadenoma. $53(88.3 \%)$ had mild hirsutism, $5(8.4 \%)$ had moderate and $2(3.3 \%)$ had severe hirsutism. The most common associated sign of hyperandrogenism in our study was seborrhoea (50\%), followed by acne (43.3\%), acanthosis nigricans (25\%) and androgenetic alopecia (18.3\%). 5 (8.3\%) had associated metabolic syndrome and all of them had PCOS.

Limitations- A small sample size and free testosterone levels were not estimated.

\section{CONCLUSION}

PCOS is the most common cause of hirsutism in this study. The term IH should be reserved only for those patients who have negative findings after a thorough investigation. Side burns, which is not included in $\mathrm{mF}-\mathrm{G}$ scoring were more seen than the involvement of upper arms. The severity of hirsutism shows no correlation with androgen levels. So all hirsute women should undergo a complete workup.

\section{KEYWORDS}

Hirsutism, Polycystic Ovary Syndrome, Modified Ferriman-Gallwey Scoring.

HOW TO CITE THIS ARTICLE: Sobhanakumari K, Reshma T. A study on clinical profile of patients with hirsutism. J. Evolution Med. Dent. Sci. 2017;6(33):2726-2729, DOI: 10.14260/Jemds/2017/588

\section{BACKGROUND}

Hirsutism is the presence of terminal hair in male like pattern in females in prepubertal and adult life. ${ }^{1}$ Hirsutism can be due to high androgen levels or by higher sensitivity of hair follicles to normal androgen levels. The causes of hirsutism include PCOS, Cushing syndrome, hyperprolactinaemia, NCCAH, idiopathic and rarely ovarian or adrenal tumours. ${ }^{2}$

Hirsutism is a reason for cosmetic embarrassment, poor self-esteem, psychological distress for women that adversely affect the quality of life. ${ }^{3}$ However, hirsutism has poorly been studied in Indian patients and there are no reported studies from South India. This study was undertaken to evaluate the clinical profile of patients with hirsutism and to detect its various causes.

Financial or Other, Competing Interest: None.

Submission 18-03-2017, Peer Review 12-04-2017,

Acceptance 17-04-2017, Published 24-04-2017.

Corresponding Author:

Dr. Kunjumani Sobhanakumari,

Harivasam, Doctors Gardens,

Gandhinagar, Kottayam- 686008,

Kerala, India.

E-mail:yemyessadeep@gmail.com

DOI: $10.14260 /$ jemds $/ 2017 / 588$

\section{MATERIALS AND METHODS}

60 consecutive hirsute patients in the age group 15 to 45 years with modified Ferriman-Gallwey (mF-G) score of 8 or more attending the Dermatology \& Endocrinology Outpatient Department of Govt. Medical College, Kottayam during the period from April 2013-October 2014 were included in the study. Pregnant or lactating women, those who received oral contraceptive pills or other anti-androgen drugs in previous three months and those who received drugs known to cause hirsutism or interfere with the hormonal studies were excluded.

After taking an informed consent, detailed history was obtained including menstrual irregularities. The menstrual patterns were defined as regular if the length of cycle was between 22 and 40 days. Family history of hirsutism was obtained. Height, weight, waist circumference (WC) (midway between iliac crest and lower margin of ribs) and blood pressure were recorded. BMI was classified according to the recommendations by WHO for Asians and is depicted in table 1. 


\begin{tabular}{|c|c|}
\hline Class & BMI $\left(\mathbf{k g} / \mathbf{m}^{\mathbf{}}\right)$ \\
\hline Underweight & $<18.5$ \\
\hline Normal & $18.5-22.9$ \\
\hline Overweight at risk & $23-24.9$ \\
\hline Obese I & $25-29.9$ \\
\hline Obese II & $\geq 30$ \\
\hline Table 1. New BMI Classification for Asians by WHO \\
\hline
\end{tabular}

All were examined for acne, seborrhoea, androgenetic alopecia (AGA), acanthosis nigricans (AN), breast atrophy, clitoromegaly, and increased muscle mass.

The degree of hirsutism was assessed using the $\mathrm{mF}-\mathrm{G}$ score in the upper lip, chin, chest, upper back, lower back, upper abdomen, lower abdomen, thighs, and upper arms and graded as 0 - none, 1- mild, 2- moderate, 3- severe, 4- frankly virile. ${ }^{1}$ Hirsutism was classified as mild (score 8-16), moderate (17-24), and severe ( $>25)$. A complete systemic examination was also performed.

Blood sampling was done after overnight fasting in the early follicular phase (second or third day of menstrual cycle). Serum total testosterone, DHEAS, 17-OHP levels were measured using enzyme-linked immunosorbent assay (ELISA). Prolactin, free tri-iodothyronine (fT3), free tetraiodothyronine (fT4), and thyroid stimulating hormone (TSH) levels were measured by chemiluminescence. Fasting lipid profile and blood glucose were also done.

Transabdominal USG was done during the follicular phase of menstrual cycle (Day 3). The USG criteria to define polycystic ovaries were presence of 12 or more follicles in each ovary, and/or increase in ovarian volume $(>10 \mathrm{~mL})$ and only one ovary fitting this definition is sufficient for diagnosis. ${ }^{2}$

- The Rotterdam 2003 criteria were used for diagnosis of PCOS. 4

According this, PCOS requires two out of three among the following for diagnosis-

- Oligo and/or anovulation.

- Clinical and/or biochemical hyperandrogenism.

- Polycystic ovaries on ultrasound in which other aetiologies must be excluded.

- Data was analysed by SPSS (Statistical Package for the Social Sciences Software) version 16.0.

\section{RESULTS}

60 clinically diagnosed cases of hirsutism were studied. Mean age in the study was $26.97 \pm 7.52$ years. Majority $(56.7 \%)$ were in the age group 15 to 25 . Mean duration of hirsutism was $10.87 \pm 6.743$ years.

The commonest cause of hirsutism was PCOS 70\% followed by IH $26.6 \%$. There was one case each of NCCAH and pituitary macroadenoma. The patient with pituitary macroadenoma had galactorrhoea, hirsutism, acromegaly and bitemporal hemianopia.

5 had history of diabetes. Of the 5 with diabetes, 4 were PCOS. 25\% had family history of hirsutism among whom $60 \%$ were PCOS and the rest had IH. 59.5\% of PCOS had irregular periods. A significant association was noted between menstrual irregularity and PCOS (P value 0.001).
The mean weight of the study group was $64.42 \mathrm{~kg}$ whereas the mean weight of PCOS group was $66.38 \mathrm{~kg}$. On comparing weight of PCOS group with others using independent sample t test, $\mathrm{p}$ value was 0.001 . The mean BMI was $25.51 \pm 3.651 \mathrm{~kg} / \mathrm{m}^{2} .31 .7 \%$ were overweight, $40 \%$ were obese I, $11.6 \%$ were obese II and the remaining had normal BMI. In those with PCOS, $90.5 \%$ had above normal BMI. P value of the association between abnormal BMI and PCOS was 0.014 . Signs of hyperandrogenism like acne in $43.3 \%$, seborrhoea in $50 \%$, AN in $25 \%$ and AGA in $18.3 \%$ were seen in the study group. The distribution of signs of hyperandrogenism in PCOS group is shown in table 2 and that of IH is shown in table 3.

\begin{tabular}{|c|c|c|}
\hline & Frequency & p Value \\
\hline Acne & $22(52.4 \%)$ & 0.031 \\
\hline Seborrhoea & $24(57.1 \%)$ & 0.091 \\
\hline AN & $12(28.6 \%)$ & 0.329 \\
\hline AGA & $8(19 \%)$ & 0.827 \\
\hline \multicolumn{2}{|c|}{ Table 2. Signs of Hyperandrogenism in PCOS } \\
\hline
\end{tabular}

\begin{tabular}{|c|c|c|}
\hline & Frequency & p Value \\
\hline Acne & $4(25 \%)$ & 0.084 \\
\hline Seborrhoea & $5(31.2 \%)$ & 0.08 \\
\hline AN & $2(12.5 \%)$ & 0.178 \\
\hline AGA & $2(12.5 \%)$ & 0.08 \\
\hline \multicolumn{2}{|c|}{ Table 3. Signs of Hyperandrogenism } \\
in Idiopathic Hirsutism \\
\hline
\end{tabular}

There were no signs of hyperandrogenism in the patient with NCCAH. The patient with acromegaly had associated androgenic alopecia, seborrhoea and acanthosis nigricans. Four patients had SAHA syndrome (Seborrhoea, acne, hirsutism, AGA). All the 4 had polycystic ovaries in USG abdomen and thus called ovarian SAHA. None had any sign of virilisation.

$53(88.3 \%)$ had mild hirsutism, 5 (8.4\%) had moderate and $2(3.3 \%)$ had severe hirsutism. The mean $\mathrm{mF}$-G score was $10.38 \pm 3.253$. The maximum score was 26.24 (40\%) had sideburns.

The frequency of sites affected is given in illustration 1.

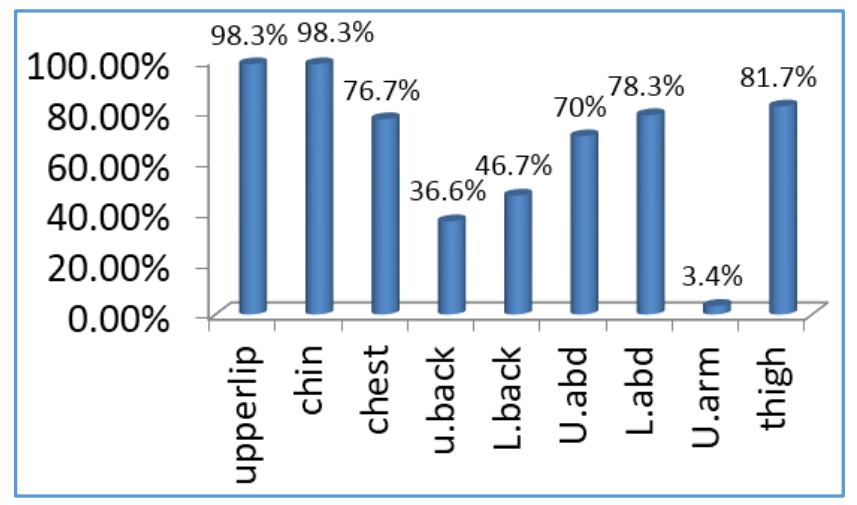

Figure 1

The mean mF-G score was highest (2.05) in upper lip and chin followed by thigh (1.62) and lower abdomen (1.35). Score was least in upper back. Severity of hirsutism in each group is presented in illustration 2 . 


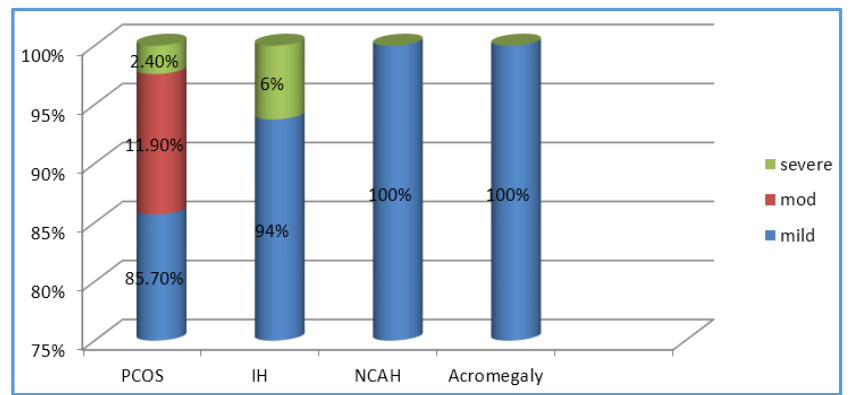

Figure 2

A two-sample t-test for comparison of mean values of $\mathrm{mF}$ $G$ score for PCOS and IH groups revealed no significant difference. No association was found between severity of hirsutism and signs of hyperandrogenism. Hormonal profile of each group is depicted in table 4 .

\begin{tabular}{|c|c|c|c|c|c|c|c|c|c|c|}
\hline \multirow[t]{2}{*}{ Group } & \multicolumn{2}{|c|}{$\begin{array}{l}\text { Testost- } \\
\text { erone }\end{array}$} & \multicolumn{2}{|c|}{ DHEAS } & \multicolumn{2}{|c|}{$\begin{array}{l}\text { Prol- } \\
\text { actin }\end{array}$} & \multicolumn{2}{|c|}{$\begin{array}{c}\text { 17- } \\
\text { OHP }\end{array}$} & \multicolumn{2}{|c|}{ TSH } \\
\hline & NL & $\uparrow$ & NL & $\uparrow$ & NL & $\uparrow$ & NL & 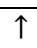 & NL & $\uparrow$ \\
\hline PCOS & 35 & 7 & 40 & 2 & 41 & 1 & 42 & 0 & 38 & 4 \\
\hline $\mathrm{IH}$ & 16 & 0 & 16 & 0 & 16 & 0 & 16 & 0 & 16 & 0 \\
\hline NCCAH & 1 & 0 & 1 & 0 & 1 & 0 & 0 & 1 & 1 & 0 \\
\hline Acromegaly & 1 & 0 & 1 & 0 & 0 & 1 & 1 & 0 & 1 & 0 \\
\hline
\end{tabular}

The patient with pituitary tumour had thrice the normal prolactin levels and raised growth hormone levels. All those with IH had normal hormonal profile. No significant association was noted between severity of hirsutism and serum total testosterone levels. $5(8.3 \%)$ had associated metabolic syndrome and all of them had PCOS ( $p$ value 0.126).

USG abdomen findings were as follows: bilateral polycystic ovaries in $30(50 \%)$, unilateral polycystic ovaries in 10 (16.7\%), normal in 17 (28.3\%) and fatty liver in 3 (5\%). Of 42 patients with PCOS, 39 (92.8\%) had polycystic ovaries in USG. The patient with pituitary tumour showed polycystic ovaries in USG. A significant association was noted between presence of polycystic ovaries and PCOS (p value 0.0001 ).

\section{DISCUSSION}

Hirsutism usually has a benign course, but may be the presenting feature of a serious underlying disease. The mean age ranges from 20.9 years to 24.18 years and mean duration ranges from 5 to 6.1 years in various studies.5,6 The duration of hirsutism in our study was higher, may be because unlike other studies a higher proportion of patients had mild hirsutism for which they were adapting their own methods of hair removal.

PCOS and IH are the two most common causes of hirsutism. The prevalence of PCOS in hirsute patients ranges from $38 \%$ to $70 \%$ and that of $\mathrm{IH}$ ranges from $15 \%$ to $60 \%$ in various studies.5,6 PCOS was the most common cause of hirsutism in our study. Earlier those patients with regular menses were taken as having idiopathic hirsutism and so the prevalence was high. But the current diagnosis of IH should be one of exclusion, in which ovulatory dysfunction, hyperandrogenaemia, and other androgen excess disorders should be ruled out. 7
The prevalence of NCCAH varies according to ethnic background and ranges from $0.38 \%$ to $7.5 \%$ in hirsute population.5,6 The patient with NCCAH presented with an ovulatory uterine bleeding and pubertal onset hirsutism like previous reports. 5,6 Our patient with pituitary macroadenoma had hyperprolactinaemia, vision defects and sudden onset hirsutism. A similar presentation was reported by Jain et al. ${ }^{8}$ In the present study, family history of hirsutism was found in $25 \%$ of whom $60 \%$ had PCOS. This supports the fact that PCOS may be inherited in a polygenic fashion. ${ }^{9}$

PCOS is the most common cause of hyperandrogenism and the raised androgen profile causes menstrual irregularities. But a history of normal menstrual cycle does not rule out PCOS, as in this study $40.5 \%$ of PCOS group had normal menses. An Iranian study also reported normal cycles in $40.4 \%$ PCOS cases. ${ }^{10}$

We noticed high proportion of obese patients unlike other studies because we have followed new classification of BMI for Asians by WHO. Also in our study, majority had PCOS which is associated with a high BMI. In PCOS the underlying insulin resistance is responsible for the increased BMI. ${ }^{11}$

SAHA syndrome was found in $9.5 \%$ of PCOS group among whom one was diabetic. Another study reported a prevalence of $17.7 \%$ of SAHA syndrome in PCOS population. ${ }^{10}$ SAHA syndrome is an acronym which stands for seborrhoea, acne, hirsutism and androgenic alopecia. It was described in 1982. It is classified into five subtypes - they are ovarian, adrenal, hyperprolactinaemic, idiopathic and HAIR-AN (hyperandrogenism, insulin resistance and acanthosis nigricans) syndrome.10 Ovarian SAHA is found to be associated with more insulin-resistant profile. We couldn't find any association between hyperandrogenism and severity of hirsutism.

Unlike other North Indian studies, we noted a lower mean mF-G score.5,6 The lower mean hirsutism score in this South Indian study may be because of genetic difference of 5-alphareductase activity in the skin.

The most commonly involved sites in our study were upper lip and chin followed by thigh as in other studies.5,6,10 Although not included in routine scoring systems, side burns were found in $40 \%$ whereas, upper arm which is a site included in $\mathrm{mF}-\mathrm{G}$ scoring was involved only in $2.4 \%$. Similar observations were done by Hassa et al also. ${ }^{12}$ This may be due to the racial dissimilarities in hair distribution. Most of the hirsutism scoring were developed initially on white population.

Hormonal workup in hirsute patients is essential to find the cause of hirsutism. Rotterdam criteria itself suggests the exclusion of other disorders like NCCAH, thyroid dysfunction, hyperprolactinaemia, neoplastic androgen secretion, druginduced androgen excess and Cushing's syndrome before diagnosing a patient as PCOS. 4 Also idiopathic hirsutism, another common cause is always the diagnosis of exclusion.

Studies show that total testosterone levels in hirsute patients can be misleading because of inherent variation in commercial testing methods. ${ }^{13}$ So estimation of free testosterone levels are more useful in diagnosis of PCOS. The proportion of PCOS patients with biochemical hyperandrogenism was lesser $(16.67 \%)$ in our patients, compared to other studies (71.43\%), may be because free testosterone level was not checked in this study. Similar to 
other studies, we also couldn't find any association between severity of hirsutism and total testosterone levels. ${ }^{7}$

In this study, DHEAS was raised in two patients with PCOS. Serum DHEAS levels may be increased (up to 8 $\mathrm{mg} / \mathrm{mL}$ ) in $50 \%$ of an ovulatory women with PCOS. So obtaining DHEAS level routinely in PCOS patients is not recommended because it does not change the diagnosis or management. ${ }^{14}$ The cause of adrenal hyperactivity in PCOS is not known. DHEAS levels above $8 \mathrm{mg} / \mathrm{mL}$ may be associated with steroidogenically active adrenal tumours, and imaging is then indicated. ${ }^{14}$

There was a single patient with NCCAH and her basal $17-$ hydroxyprogesterone level was higher than $8 \mathrm{ng} / \mathrm{mL}$. In pituitary tumour, growth hormone (GH) and prolactin levels are raised. This leads to decrease of sex hormone binding globulin levels which in turn results in increased free testosterone. ${ }^{15}$ Also, GH increases IGF-1 (Insulin like growth factor-1), increases ovarian androgen production and acanthosis nigricans as seen in our patient. ${ }^{15}$

Metabolic syndrome was found in $11.9 \%$ of PCOS group. The prevalence varies from $12.5 \%$ to $47.3 \%$ in various studies. ${ }^{11}$ Differences in ethnicity, environment, and diet can alter the frequency of PCOS-related metabolic abnormalities. The prevalence of metabolic syndrome in PCOS patients is almost twice as great as in the general population of women, raising the cardiovascular risk 7 times. ${ }^{11}$

Polycystic ovaries in USG was noted in $66.7 \%$. The presence of polycystic ovaries in USG is not specific for PCOS as they emerge in a nonspecific fashion when a state of anovulation persists for a longtime. ${ }^{14}$ So all the investigations (biochemical, sonological) should be done in the specified time in a hirsute of any severity to find out the exact aetiology.

\section{Limitations}

Free testosterone levels were not estimated in this study. Also, here the sample size is small, large population based studies are to be done to arrive at definite conclusions.

\section{CONCLUSION}

In conclusion, the commonest cause of hirsutism in our study was PCOS. The term idiopathic hirsutism is probably overused in the diagnosis of hirsutism, and should therefore be reserved only for those patients who have negative findings after a thorough investigation. As side burns, which is not included in $\mathrm{mF}-\mathrm{G}$ scoring were seen in more patients than the involvement of upper arms, we propose that hirsutism scoring should be population specific. The severity of hirsutism shows no correlation with androgen levels. In fact even hyperandrogenaemic patients with PCOS or NCCAH may not have severe hirsutism and, in contrast, women with idiopathic hirsutism with entirely normal circulating androgen concentrations may present with severe hirsutism. So irrespective of severity, all hirsute women should undergo a complete workup and need proper followup. We believe such a workup will unravel a cause for the hirsutism in most if not all women afflicted with this problem. This will help in appropriate management of these desperate patients.

\section{ACKNOWLEDGMENTS}

We express our heartfelt gratitude to the Department of Endocrinology, Government Medical College, Kottayam for their help and support to conduct this study.

\section{REFERENCES}

[1] Ferriman D, Gallwey JD. Clinical assessment of body hair growth in women. J Clin Endocrinol Metab 1961;21(11):1440-7.

[2] Azziz R, Carmina E, Dewailly D, et al. The Androgen Excess and PCOS Society criteria for the polycystic ovary syndrome: the complete task force report. Fertil Steril 2009;91(2):456-88.

[3] WHO/IASO/IOTF. The Asia-Pacific perspective: redefining obesity and its treatment. Health Communications Australia Pty Ltd., 2000.

[4] Rotterdam ESHRE/ASRM-Sponsored PCOS Consensus Workshop Group. Revised 2003 consensus on diagnostic criteria and long-term health risks related to polycystic ovary syndrome. Fertil Steril 2004;81(1):19-25.

[5] Sharma D, Shanker V, Tegta G, et al. Clinicoinvestigative profile of patients of hirsutism in a tertiary level institution. Int J Trichology 2012;4(2): 69-74.

[6] Chhabra S, Gautam RK, Kulshreshtha B, et al. Hirsutism: a clinico-investigative study. Int J Trichol 2012;4(4):246-50.

[7] Azziz R, Waggoner WT, Ochoa T, et al. Idiopathic hirsutism: an uncommon cause of hirsutism in Alabama. Fertil Steril 1998;70(2):274-8.

[8] Jain R, Dutta D, Shivaprasad KS, et al. Acromegaly presenting as hirsuitism: uncommon sinister aetiology of a common clinical sign. Ind J Endocr Metab 2012;16(Suppl 2):S297-S9.

[9] Urbanek M, Legro RS, Driscoll RA, et al. Thirty-seven candidate genes for polycystic ovary syndrome: strongest evidence for linkage is with follistatin. Proc Natl Acad Sci USA 1999;96(15):8573-8.

[10] Ansarin H, Azia-Jalali MH, Rasi A, et al. Clinical presentation and etiologic factors of hirsutism in premenopausal Iranian women. Arch Iranian Med 2007;10(1):7-13.

[11] Neves EM, Fonseca AM, Bagnoli VR, et al. Polycystic ovary syndrome: correlation between phenotypes and metabolic syndrome. J Steroids Hormon Sci 2014;5(2):132-4.

[12] Hassa H, Tanir HM, Yildirim A, et al. The hirsutism scoring system should be population specific. Fertil Steril 2005;84(3):778-80.

[13] Boots LR, Potter S, Potter D, et al. Measurement of total serum testosterone levels using commercially available kits: high degree of kit variability. Fertil Steril 1998;69(2):286-92.

[14] Bulun SE, Adashi EY. The physiology and pathology of female reproductive axis. In: Kronenberg HM, Melmed $\mathrm{S}$, Polonsky KS, et al. eds. William textbook of endocrinology. $11^{\text {th }}$ edn. Philadelphia: Saunders 2008:546-63.

[15] Kaltsas GA, Mukherjee JJ, Jenkins PJ, et al. Menstrual irregularity in women with acromegaly. J Clin Endocrinol Metab 1999;84(8):2731-5. 